\title{
Crystal structure of aluminum germanium manganese (72.0:26.3:57.7), $\mathrm{Al}_{72.0} \mathrm{Ge}_{26.3} \mathrm{Mn}_{57.7}$
}

\author{
Kazumasa Sugiyama*,I, Hironori Sato ${ }^{\mathrm{I}}$, Yoshihiko Yokoyama ${ }^{\mathrm{II}}$, Kenji Hiraga ${ }^{\mathrm{II}}$ and Kenji Yoza ${ }^{\mathrm{III}}$ \\ I University of Tokyo, Graduate School of Science, Department of Earth and Planetary Science, 7-3-1 Hongo, Bunkyo-ku, Tokyo 113-0033, Japan \\ II Tohoku University, Institute for Materials Research, 2-1-1 Katahira, Aoba-Ku, Sendai 980-8577, Japan \\ III Bruker AXS K.K., 3-9-A Moriya, Kanagawa-ku, Yokohama 221-0022, Japan
}

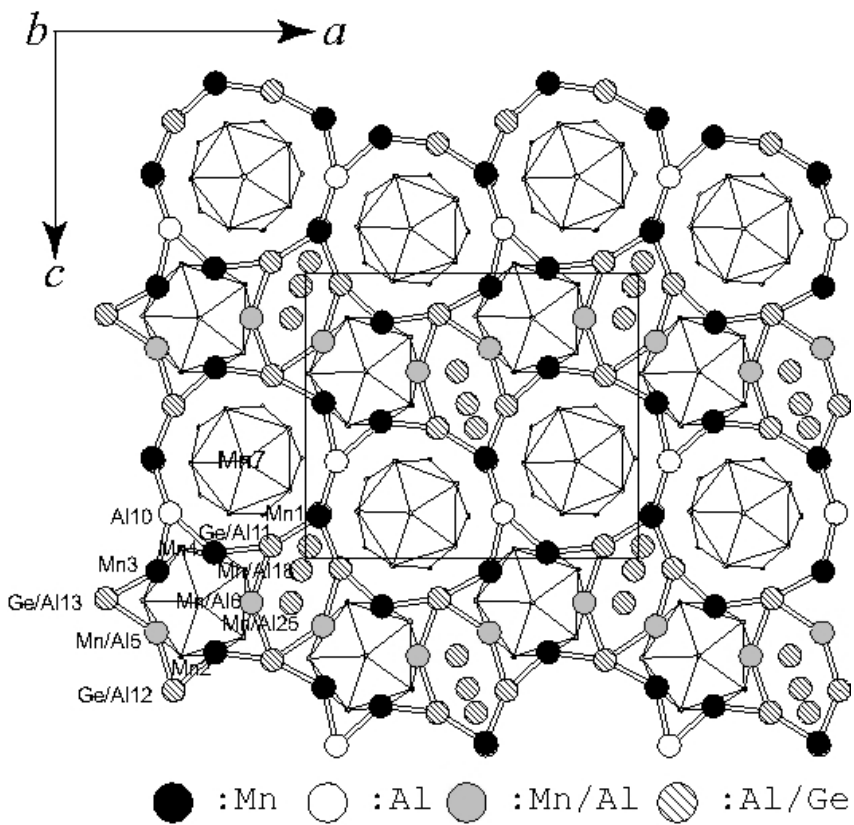

\section{Abstract}

$\mathrm{Al}_{72.0} \mathrm{Ge}_{26.3} \mathrm{Mn}_{57.7}$, orthorhombic, Pnma (no. 62), $a=14.516(2) \AA, b=12.484(2) \AA, c=12.405(2) \AA$, $V=2248.0 \AA^{3}, Z=1, R_{\mathrm{gt}}(F)=0.036, w R_{\mathrm{ref}}\left(F^{2}\right)=0.108$, $T=297 \mathrm{~K}$

\section{Source of material}

A needle-like crystal was picked up from a master ingot with nominal composition of $\mathrm{Al}_{40} \mathrm{Mn}_{35} \mathrm{Ge}_{25}$, which was prepared by arc-melting the mixture of pure $\mathrm{Al}, \mathrm{Mn}$ and $\mathrm{Ge}$ in an argon atmosphere. The chemical composition of the title compound $\mathrm{Al}_{46.1} \mathrm{Mn}_{37.0} \mathrm{Ge}_{16.9}$ was determined by the electron probe microanalysis. Another crystalline phase, $\mathrm{Al}_{34.5} \mathrm{Mn}_{33.7} \mathrm{Ge}_{31.8}$, which is frequently discussed as a magnetic ternary AlMnGe alloy [1], was also appeared in the present sample.

\section{Discussion}

Since the discovery of quasicrystals in rapidly solidified Al-rich manganese alloys [1,2], the so-called approximant structures have been used for providing structural models of quasicrystals. In particular, the crystal structure of $\mathrm{Al}_{3} \mathrm{Mn}$ with a pentagonal columnar structure serves an essential idea for the structure of decagonal quasicrystal with a period of about $12 \AA$ [3-6]. Mag-

\footnotetext{
* Correspondence author (e-mail: kazumasa@imr.tohoku.ac.jp)
}

netic properties for quasicrystals were extensively studied in a variety of Al-Mn based quasicrystals and Mn-Mn interactions were counted as one of the possible origins for their properties [7-10]. Since an $\mathrm{Al}_{3} \mathrm{Mn}$-type approximant for the Al-Mn-Ge decagonal quasicrystal also indicated a large magnetization anisotropy [9], we carried out the structural analysis of the $\mathrm{Al}_{3} \mathrm{Mn}$-type approximant in this ternary alloy system.

The diffraction patterns of the title compound are quite similar to those of the typical $\mathrm{Al}_{3} \mathrm{Mn}$ phase including the systematic absences of $k+l=2 n$ for the $0 k l$ plane and $h=2 n+1$ for the $h k 0$ plane [3-6]. These results readily lead the space group of Pnma or $P n 2{ }_{1} a$. The converged parameters in the non-centrosymmetric $P n 2_{1} a$ model serve no significant differences from those in the Pnma model, and present analysis selects the centrosymmetric space group Pnma. It is usually difficult to determine the distribution of three elements by using the diffraction data alone. Nevertheless, we can obtain here one of the most feasible distribution models as follows. The electron density distribution and corresponding first nearest neighbor distances indicate that $\mathrm{Mn} 1, \mathrm{Mn} 2$, Mn3, Mn4, Mn/Al5, Mn/Al6 and Mn7 are almost fully occupied by $\mathrm{Mn}$. The highest electron density was observed at Ge/Al25 and $\mathrm{Ge}$ is suggested to reside here. $\mathrm{Mn} / \mathrm{Al} 8, \mathrm{Mn} 9, \mathrm{Mn} / \mathrm{Al} 15$ and $\mathrm{Mn} / \mathrm{Al} 16$ are coordinated by Ge/Al25 with distances from $2.51 \AA$ to $2.58 \AA$, suggesting the preference of the smallest $\mathrm{Mn}$ at these sites, again. The fundamental preference of $\mathrm{Al}$ at remaining thirteen sites was revealed and interatomic distances among them lie over $2.66 \AA$, except for $d(\mathrm{Al} / \mathrm{Ge} 18-\mathrm{Al} / \mathrm{Ge} 18)=2.276(3) \AA$. Taking into account such features, the structure of the title compound was refined starting from the structural model of $\mathrm{Al}_{3} \mathrm{Mn}$ with chemically disordered eleven $\mathrm{Mn} / \mathrm{Al}$ sites and fourteen $\mathrm{Al} / \mathrm{Ge}$ sites. Several least-squares iterations revealed the chemically ordered sites of Mn1, Mn2, Mn3, Mn4, Mn7, Mn9, Al10 and Al14. Final results include the restrictions of the chemical composition ratio determined by the electron probe micro analysis. Since the short distance for the Al18-Al18 pair is well reproduced in the $\mathrm{Al}_{3} \mathrm{Mn}$ structure [3-5], the unusual $\mathrm{Al} / \mathrm{Ge} 18-\mathrm{Al} / \mathrm{Ge} 18$ distance for the title compound may be attributed to the partial distribution of Ge together with small coordination polyhedra of Mn1 at both ends of this pair.

The crystal structure of the title compound consists of pentagonal columnar arrangements running parallel to the $b$ axis. The atomic distribution of such pentagonal columns can be readily implied in order to reproduce the structure of decagonal quasicrystals. A number of $\mathrm{Mn}-\mathrm{Mn}$ pairs with the distance less than $2.8 \AA$ are located on the mirror plane perpendicular to the $b$ axis and a pentagonal atomic arrangement of $\mathrm{Mn} 3-\mathrm{Mn} 4-\mathrm{Mn} / \mathrm{Al} 6-\mathrm{Mn} 2-\mathrm{Mn} / \mathrm{Al} 5$ could be found, in particular. It is quite natural to attribute such a Mn pentagon to the origin of the easy magnetization axis in the (010) plane of the title compound and quasiperiodic plane of the corresponding decagonal phase. 
Table 1. Data collection and handling.

$\begin{array}{ll}\text { Crystal: } & \text { black, irregular, size } 0.03 \times 0.06 \times 0.09 \mathrm{~mm} \\ \text { Wavelength: } & \text { Mo } K_{\alpha} \text { radiation }(0.71069 \AA) \\ \mu: & 171.19 \mathrm{~cm}^{-1} \\ \text { Diffractometer, scan mode: } & \text { Bruker AXS SMART APEX-II CCD, } \psi / \omega \\ 2 \theta_{\max }: & 55.68^{\circ} \\ N(h k l)_{\text {measured }}, N(h k l)_{\text {unique }}: & 12674,2786 \\ \text { Criterion for } I_{\mathrm{obs}}, N(h k l)_{\mathrm{gt}}: & I_{\mathrm{obs}}>2 \sigma\left(I_{\mathrm{obs}}\right), 2120 \\ N(\text { param })_{\text {refined }} & 211 \\ \text { Programs: } & \text { SHELXL-97 [12], ATOMS [13] }\end{array}$

Table 2. Atomic coordinates and displacement parameters (in $\AA^{2}$ ).

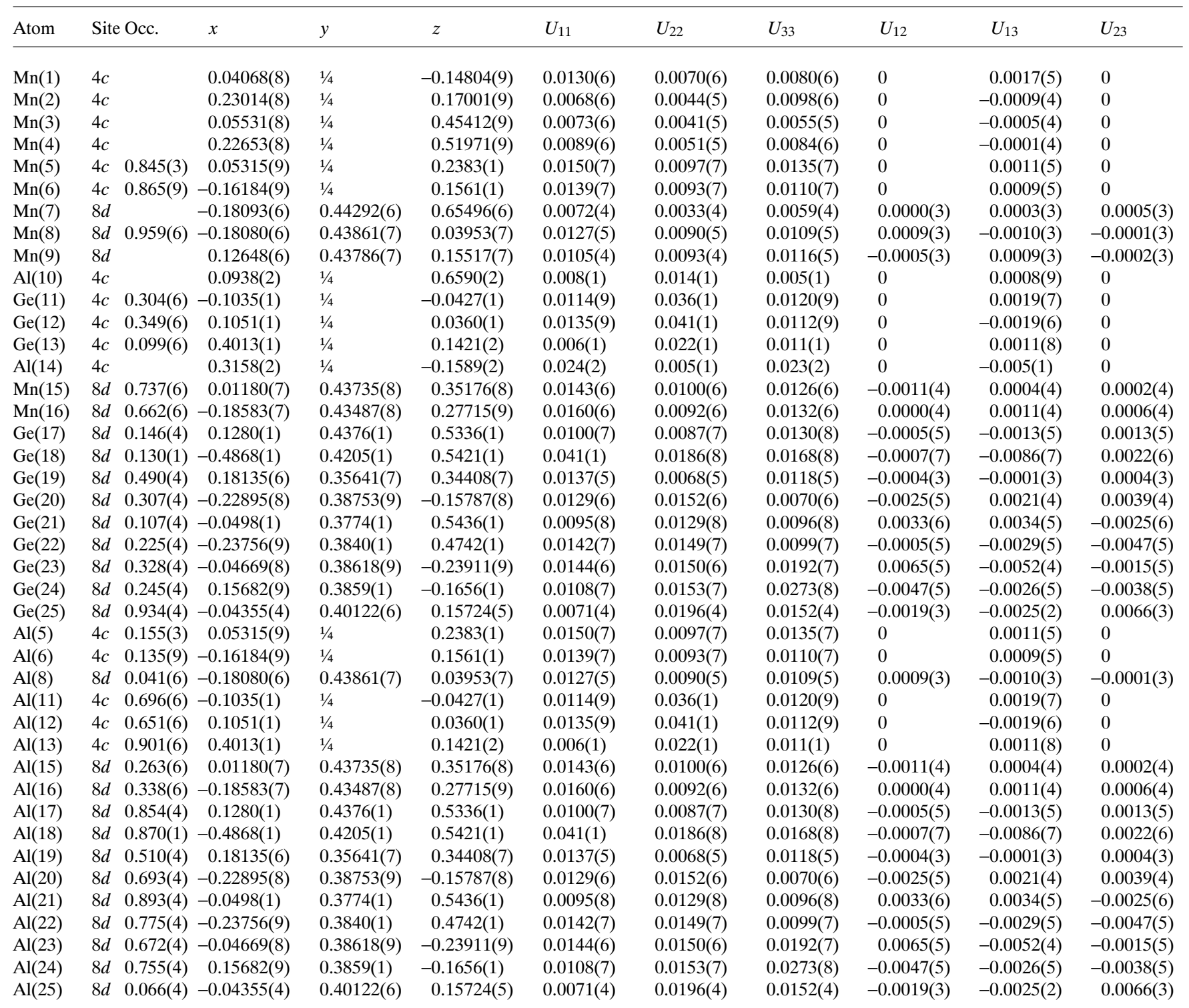

Acknowledgments. The authors gratefully acknowledge the financial support of Grant-in-Aid for Scientific Research (B) (grant no. 14340161) from the Ministry of Education, Culture, Sports, Science and Technology (MEXT).

\section{References}

1. Murthy, N. S.; Begum, R. J.; Somanathan, C. S.; Murthy, M. R. L. N.: Magnetic structure of MnAlGe. J. Appl. Phys. 40 (1969) 1870-1871.

2. Shechtman, L.; Blech, I.; Gratias, D.; Cahn, J. W.: Metallic phase with long-range orientational order and no translational symmetry. Phys. Rev. Lett. 53 (1984) 1951-1953.
3. Bendersky, L: Quasicrystal with one-dimensional translational symmetry and a tenfold rotation axis. Phys. Rev. Lett. 55 (1985) 1461-1463.

4. Hiraga, K.; Kaneko, M.; Matsuo, Y.; Hashimoto, S.: The structure of $\mathrm{Al}_{3} \mathrm{Mn}$ : close relationship to decagonal quasicrystal. Philos. Mag. B67 (1993) 193-205. 
5. Shi, N. C.; Li, X. Z.; Ma, Z. S.; Kuo, K. H.: Crystalline phases related to a decagonal quasicrystal. I. A single-crystal X-ray diffraction study of the orthorhombic $\mathrm{Al}_{3} \mathrm{Mn}$ phase. Acta Crystallogr. B50 (1994) 22-30.

6. Pavlyuk, V. V.; Yanson, T. I.; Bodak, O. I.: Structure refinement of orthorhombic $\mathrm{MnAl}_{3}$. Acta Crystallogr. C51 (1995) 792-794.

7. Matsuo, Y.; Kaneko, M.; Yamanoi, T.; Kaji, N.; Sugiyama, K.; Hiraga, K.: The structure of an $\mathrm{Al}_{3} \mathrm{Mn}$-type $\mathrm{Al}_{3}(\mathrm{Mn}, \mathrm{Pd})$ crystal studied by singlecrystal X-ray diffraction analysis. Philos. Mag. Lett. 76 (1997) 357-362.

8. Yokoyama, Y.; Inoue, A.: Ferromagnetic Mn-based decagonal alloys at room temperature. Jpn. J. Appl. Phys. 35 (1996) 3533-3534.

9. Tsai, A. P.; Inoue, A.; Masumoto. T.; Kataoka, N.: Al-Ge-Mn and Al-CuGe-Mn quasicrystals with coercivity at room temperature. Jpn. J. Appl. Phys. 27 (1988) L2252-L2255.
10. Yokoyama, Y.; Inoue, A.; Note, R.; Kaneko, T.: Quasi- and approximantcrystalline Al-Mn-Ge alloys with high coercive force. Mater. Trans. 39 (1988) 237-244

11. Shinohara, T.; Yokoyama, Y.; Saito, M.; Inoue, A.; Masumoto, T.: Nuclear magnetic resonance study of new strong magnetism in quasicrystalline Al-Pd-Mn-B alloy. Mater. Sci. Eng. A181/182 (1994) 798-800.

12. Sheldrick, G. M.: SHELXL-97. Program for the Refinement of Crystal Structures. University of Göttingen, Germany 1997.

13. Dowty, E.: ATOMS. A Complete Program for Displaying Atomic Structures. Version 6.2. Shape Software, Kingsport, Tennessee, USA 2005. 